\title{
2019 Conference of the Society for Business Ethics Call For Submissions and Reviewers
}

The Colonnade Hotel

Boston, Massachusetts (USA)

August 9-11, 2019

\section{Submission Deadline: February 15, 2019}

The Society for Business Ethics (SBE) welcomes scholarly submissions and reviewers for its 2019 Annual Conference. Please upload your submissions at the website: https://mc.manuscriptcentral. com/sbeconference. Only electronic submissions to this website, in PDF format, will be considered. If you have any questions or suggestions regarding the program, please contact the Program Chair, Jeff Frooman: frooman@unb.ca. Information regarding the conference registration and hotel will be available at the SBE website: https://www.sbeonline.org/

We accept six types of submissions, as follows:

1) Paper Submissions: Papers should be double-spaced and use 12-point Times New Roman (or similar) type, and should be no longer than 30 pages (double-spaced). The manuscript itself must be suitable for blind review (prepared without a title page or any author identifying information in the text or notes). The paper should be uploaded as a PDF file. When submitting online, authors will be requested to provide a title, an abstract of no more than 250 words, five keywords, and at least three submission codes (listed below) that summarize the submission's topic areas and methodologies.

2) Agora Submissions: In classical Greece, the agora was a public meeting place that often served as a market and sometimes served as a forum for intellectual discourse with an audience of whomever was interested. Because business ethics is a topic with important implications for management practice, we welcome submissions that are intended to explain business ethics concepts clearly and winsomely for popular audiences. These submissions ought to have the same attributes as those listed for category 1 above; however, they will be evaluated on the basis of their critical engagement with the relevant literature, their clarity, and their practical implications. Moreover, accepted Agora submissions will be presented at a special concurrent session in which presenters are encouraged to abandon the usual conventions of academic paper presentations, and instead make presentations that could readily appeal to popular audiences . . . perhaps, drawing inspiration from TEDx talks or other similarly accessible styles.

3) Review Submissions: SBE invites proposals for manuscripts that provide comprehensive and insightful scholarly surveys of topics relevant to business ethics research. Review papers summarize the existing scholarly literature on a particular subject and utilize that review as a basis for making a novel theoretical contribution that advances our understanding of the subject and provides valuable directions for future research on the topic. Work from both the social sciences and the humanities is welcome. Proposals should have the attributes listed for category 1 above, but be no more than ten pages long (double-spaced), not including references. Please limit references to two pages, single-spaced, highlighting the most significant works in the topic area you propose to survey. The proposed review manuscript must address an important and substantial area of research, integrating a wide range of research on that topic in a way that makes a clear contribution to the advancement of theory and research relevant to business ethics. The contribution to advancing theory and research is essential; proposals that merely summarize existing research will not be accepted. The proposal should be clearly organized, well-argued, and should engage the relevant existing research well. The proposal should also 
be defined precisely enough that the reviewers will be able to judge the likelihood that you can develop a manuscript of appropriate length and scope that realizes the contribution envisioned in the proposal.

4) Panel Submissions: These are thematically organized sessions intended to advance theoretical understanding, analyze the implications of recent business activity or public policy developments, or provide pedagogical guidance. Submissions should be in the range of three to six pages and should identify the chair and all proposed participants by name, address, phone number, and email address. Panel proposals should include a title, a clear statement indicating the rationale for the panel, the format to be used, and the contribution of each panelist. The proposal should be uploaded as a PDF file. When submitting online, the panel proposer will be requested to provide an abstract of no more than 250 words.

5) Workshop Submissions: Workshops are interactive sessions where the attendees are participants and typically focus on scholarly development or pedagogy, although other types of submissions are welcome. Proposals should include a title and a clear statement indicating the rationale for the workshop, why your research and/or expertise qualify you to give this workshop, and how you would run the workshop. There should be no more than three workshop leaders. The proposal should be uploaded as a PDF file. When submitting online, the workshop leader will be requested to provide an abstract of no more than 250 words.

6) Emerging Scholars Submissions: $\mathrm{PhD}$ candidates may submit an abstract of between 750 and 1,000 words describing a research project in business ethics or related domains. Abstracts should include a short list of relevant citations and include a cover page with full contact information and affiliation. In addition to being able to present their ideas in a paper session, students whose papers are accepted will be invited to attend a half-day workshop with senior SBE mentors. Although a PhD student may be an Emerging Scholar more than once, priority is given to those who have not previously been accepted into the SBE conference program as an Emerging Scholar. The abstract should be uploaded as a PDF file.

Multiple Submissions: In an effort to be inclusive, and facilitate the participation of as many scholars as possible in the conference, individuals submitting paper proposals will be limited to one paper submission or one emerging scholar submission, but not both. (However, a PhD candidate who has submitted an abstract as an emerging scholar may be a co-author with another established scholar as lead author.) Scholars may also be included in up to two panel or workshop submissions for a total of three submissions. These rules may be relaxed if there is available space and a sufficient diversity of scholarly perspectives is represented on the program. These rules will not prevent any program participant from also serving as a session chair appointed by the Program Committee.

Reviewers: Individuals who submit paper, panel, and workshop proposals will automatically be considered by the Program Committee to be eligible peer reviewers for other submissions within their areas of expertise. We welcome and strongly encourage others to volunteer to serve as a peer reviewer by following the "Create An Account" tab at the submissions website: https://mc.manuscriptcentral.com/sbeconference. Authors and reviewers who have participated in any SBE Annual Conference since 2015 need not create a new account to be considered a reviewer for the 2019 Annual Conference.

Submission Codes: To facilitate matching submissions with reviewers, paper submissions should also include a three-letter code according to the following (list at least one number from Theoretical Perspectives, Application Contexts, and Methodologies [e.g., T-6; A-4; M-1]). Submissions will be considered that do not "fit" any of these categories. 


\section{Theoretical Perspectives}

T-1 Accounting theory

T-2 Comparative ethics/Pluralism

T-3 Consequentialism

T-4 CSR/corporate citizenship theory

T-5 Critical theory

T-6 Deontological ethics

T-7 Economics (micro or macro)

T-8 Feminism/Care ethics

T-9 Finance Theory

T-10 Legal studies

T-11 Marketing Theory

T-12 Organizational behavior/psychology

T-13 Organizational theory/sociology

T-14 Pedagogy

T-15 Political theory

T-16 Religion/Theology

T-17 Social contract theory

T-18 Stakeholder theory

T-19 Strategic management

T-20 Virtue ethics

T-21 Other

\section{Application Contexts}

A-1 Accounting

A-2 Business and human rights

A-3 Competitive practices

A-4 Corruption/Bribery

A-5 Corporate communications/

Transparency/Media relations

A-6 Corporate governance

A-7 Corporate reporting/accountability programs

A-8 Corporate social responsibility

A-9 Corporate/Industry self-regulation
A-10 Distributive/Procedural justice

A-11 Employment and labor issues

A-12 Entrepreneurship

A-13 Ethical decision making and behavior

A-14 Environmental sustainability

A-15 Finance

A-16 International business/ TNCs/ Globalization

A-17 Industry-specifc issues

A-18 Information technology/Internet

A-19 Legal and Regulatory issues

A-20 Marketing/Advertising

A-21 Organizational culture

A-22 Organizational ethics programs/ initiatives

A-23 Small- and medium-sized enterprises

A-24 Social/Ethical investing

A-25 Spirituality/Religion in workplace

A-26 Teaching/Curriculum

A-27 Stakeholder relations

A-28 Other

\section{Methodologies}

M-1 Analytical/Conceptual/Theoretical

M-2 Case study (single case or comparative cases)

M-3 Historical

M-4 Qualitative (e.g., interview, participant-observer, ethnographic)

M-5 Quantitative data (experimental)

M-6 Quantitative data (feld)

M-7 Quantitative data (measure development)

M-8 Other 